\title{
Etiopathogenic and Clinical Aspects in Inflammatory Bowel Disease - Literature Review
} \author{
Ciprian Rezus ${ }^{1,2}$ \\ 1 "Grigore T. Popa" University of Medicine and Pharmacy, Iași, Romania \\ 2 3rd Internal Medicine Clinic, "Sf. Spiridon" Emergency County Clinical Hospital, Iași, Romania \\ 3 Rehabilitation Hospital, Neurology Clinic, Iași, Romania \\ 4 Rehabilitation Hospital, Rheumatology Clinic, lași, Romania
}

Petronela Nicoleta Seritean Isac',2, Diana Popescu1,2, Tudor Marcel Genes ${ }^{1,3}$, Minerva Codruta Badescu1,2, loana Irina Rezus¹, Nicoleta Dima1,2, Ana Roxana Rusu Ganceanu1,2, Elena Rezus ${ }^{1,4}$,

\section{CORRESPONDENCE \\ Petronela Nicoleta Seritean Isac \\ Str. Universității nr. 16 \\ 700115 lași, Romania \\ Tel: +40 232301615 \\ E-mail: isac_petronela@yahoo.com}

\section{ARTICLE HISTORY}

Received: July 19, 2021

Accepted: August 30, 2021

Diana Popescu • Str. Universității nr. 16, 700115 lași Romania. Tel: +40 742058 924, E-mail: dr.popescu. diana@gmail.com

Tudor Marcel Genes • Str. Universității nr. 16, 700115 Iași, Romania. Tel: +40 232301 615, E-mail: genestudor@yahoo.com

Minerva Codruta Badescu • Str. Universității nr. 16 700115 lași, Romania. Tel: +40 742963 454, E-mail codruta.badescu@gmail.com

loana Irina Rezus • Str. Universității nr. 16, 700115 lași, Romania. Tel: +40 232301 615, E-mail: ioanairinarezus@yahoo.co.uk

Nicoleta Dima • Str. Universității nr. 16, 700115 lași, Romania. Tel: +40 740322 218, E-mail: nicoleta. dima@umfiasi.ro

Ana Roxana Rusu Ganceanu • Str. Universității nr. 16 700115 lași, Romania. Tel: +40 756582 044, E-mail: ana.ganceanu-rusu@umfiasi.ro

Elena Rezus • Str. Universității nr. 16, 700115 lași, Romania. Tel: +40 232301615 , E-mail: elena_rezus@ yahoo.com

Ciprian Rezus • Str. Universității nr. 16, 700115 lași, Romania. Tel: +40 232301 615, E-mail: ciprianrezus@ yahoo.com

\begin{abstract}
Inflammatory bowel disease (IBD) is a chronic inflammatory condition which encompasses Crohn's disease and ulcerative colitis. IBD does not only affect the gastrointestinal system, but also associates many extraintestinal complications that can affect almost any organ. A large number of patients may have these complications before or after the diagnosis of IBD. Early diagnosis and management of these complications involves a multidisciplinary team and contributes to decrease patient morbidity and mortality, but also to increase the quality of life. The purpose of this extensive literature review is to present systematically and comprehensively the latest data on the extraintestinal manifestations of IBD, and to draw clinicians' attention to the fact that this condition can have extradigestive manifestations that can be misleading and delay the diagnosis.
\end{abstract}

Keywords: inflammatory bowel disease, ulcerative colitis, Crohn's disease, extraintestinal manifestations

\section{INTRODUCTION}

Inflammatory bowel disease (IBD) includes two clinical entities: Crohn's disease (CD) and ulcerative colitis (UC), both characterized by a chronic inflammation of the gastrointestinal tract, whose frequent signs and symptoms include abdominal pain, diarrhea and bloody stools. While UC is caused by a continuous inflammation of the colonic mucosa and submucosa, in CD the inflammation is full-thickness, discontinuous, and involves the entire digestive tract. ${ }^{1}$ IBDs are commonly linked with the occurrence of extraintestinal manifestations (EIMs). These can affect almost any organ system, thus contributing to increased morbidity in patients with IBD. ${ }^{2}$ 
The pathophysiology of the disease has not been fully elucidated yet. The major determinants of IBD are genetic susceptibility, intestinal flora, lifestyle, and the immune system. ${ }^{1}$ The pathogenesis of EIMs in IBD is unclear, but genetic factors and the immune system are thought to be involved. The immune response triggered by the gastrointestinal mucosa can affect extraintestinal areas due to common epitopes. Thus, translocated bacteria will trigger an adaptive immune response, unable to differentiate between bacterial or joint and skin epitopes. ${ }^{3}$

It has been shown that genetic susceptibility plays a major role. For instance, EIMs in CD occur more often in subjects that present HLA-A2, HLA-DP1, and HLA-DQW5 patterns. In UC, EIMs are more often present in subjects with HLA-DR103 haplotype. An increased probability for primary sclerosing cholangitis (PSC) in UC has been specifically associated with HLA-B8/D33. ${ }^{2}$

Short-chain fatty acids (SCFAs) create favorable conditions for resistance to pathogenic bacteria and prevent colitis, thus contributing to the maintenance of intestinal homeostasis. Lower levels of SCFA were found in patients with IBD and psoriatic arthritis. ${ }^{4}$ Long-chain fatty acids (LCFAs) have a reciprocal relationship with IBD. Along with economic development, the morbidity of IBD has shown a significant increase, to a certain extent owed to eating habits (long-chain polyunsaturated omega-6/omega-3 fatty acids [PUFAs] and an abundance of saturated fats). Moreover, LCFAs are also involved in modulating intestinal lesions. ${ }^{5}$

\section{ARTICULAR MANIFESTATIONS}

Inflammatory arthropathies are the most common EIMs of subjects with IBD. ${ }^{4}$ They are classified into axial and peripheral spondylarthritis, with a prevalence of 5-20\% and $3-25 \%$, respectively. ${ }^{6}$ Peripheral arthropathies that occur as EIMs in patients with inflammatory disorders of the digestive tract are separated into two categories. Type I arthropathy, which is pauciarticular or oligoarticular, affects maximum 5 large articular areas and is characterized by an acute, asymmetrical, and migratory pattern. It is associated with HLA-B27, HLA-B35, and HLA-DR103 haplotypes. The symptoms persist for less than 10 weeks, are self-limiting, do not cause permanent destruction of the joint, and are correlated with the intestinal activity. ${ }^{7}$ Type II arthropathy has a polyarticular pattern and may involve 5 or more small joints. The symptoms can persist for months or years and are independent of the disease course. The second type is associated with HLA-B44. ${ }^{7}$
Axial arthropathies are independent of the disease activity. The most common symptoms are back pain and morning stiffness, and may be present before the intestinal symptoms of IBD.?

Patients with IBD may experience decreased bone mineral density due to changes in absorption caused by multiple factors: intestinal resections, mineral malabsorption and vitamin D deficiency, corticosteroid treatment, and microbiota interaction. Osteoporosis is characterized by decreased bone mass and deterioration of bone microarchitecture, thus causing an increase in bone fragility and in the risk of fractures. ${ }^{8}$ Alendronate and risedronate are the most widely used therapies in the prevention of vertebral and non-vertebral fractures, with long established efficacy. ${ }^{8}$

\section{MUCOSAL SKIN MANIFESTATIONS}

Mucocutaneous manifestations in IBD are common and cover a wide spectrum of manifestations that range from mild to severe and even debilitating. ${ }^{9}$ The prevalence of these lesions is $10-15 \%$. The most frequent mucocutaneous clinical manifestations in IBD are erythema nodosum and pyoderma gangrenosum. Rare manifestations are psoriasis and Sweet's syndrome. ${ }^{7}$

Erythema nodosum is the most common cutaneous manifestation of IBD, with a prevalence of $3-10 \%$ in patients with UC and $4-15 \%$ in patients with CD. It occurs more frequently in women and in patients with HLA-B27 haplotype. Erythema nodosum is characterized by the presence of symmetrical, erythematous, warm, painful, non-ulcerative nodules, with dimensions between 1-5 $\mathrm{cm}$, located mainly on the extensor surfaces of the lower limbs. ${ }^{10}$

Pyoderma gangrenosum is a severe cutaneous manifestation, with a prevalence of $1-2 \%$ in patients with IBD, being more frequently associated with UC. This condition can be expressed before, during, or after the onset of IBD, but it can be independent also. More frequently, it affects women and patients with a family history of UC. ${ }^{10} \mathrm{Al}-$ though many forms of manifestation have been described (ulcerative, bullous, pustular, vegetative, drug-induced, postoperative, and peristomal), the most common types in IBD are ulcerative and pustular. The initial lesion presents papules, pustules, or nodules with violaceous, irregular edges, which subsequently evolve towards painful ulceration. ${ }^{10}$

Sweet's syndrome is an acute febrile neutrophilic dermatosis, rarely associated with IBD. The prevalence is higher in patients with $\mathrm{CD}$ than in those with UC and among women aged $30-50$ years. ${ }^{9}$ This syndrome is char- 
acterized by the presence of papules, nodules, and erythematous plaques with a tendency to confluence, located especially on the face, neck, and upper limbs. ${ }^{10}$ Sweet's syndrome can affect not only the skin but also other organs such as the lungs, muscles, joints, liver, kidneys, and eyes. ${ }^{9}$

\section{ORAL MANIFESTATIONS}

Oral manifestations in IBD are specific or nonspecific and may precede or coincide with the diagnosis. The most common is aphthous stomatitis with a prevalence between $0.7 \%$ and $20 \%$. It is characterized by the presence of small, painful ulcers surrounded by an erythematous halo. In patients with IBD, the lesions may be secondary to iron, zinc, or vitamin B12 deficiency, or IBD therapy. ${ }^{11}$ The oral manifestation secondary to mesalazine and sulfasalazine treatment is oral lichen planus, ${ }^{12}$ and correlated with the administration of infliximab are oral ulcerations. ${ }^{11}$

\section{EYE MANIFESTATIONS}

About $2-5 \%$ of patients with IBD have ocular symptoms. They arise more often in subjects with CD (3.5-6\%) compared to UC $(1.6-4.6 \%)^{7}$ and are represented by episcleritis, scleritis, uveitis, and conjunctivitis. ${ }^{2}$ Besides the ocular manifestations due to IBD, ocular complications secondary to specific therapy can also be observed. Corticosteroids can cause cataract and open-angle glaucoma. Cyclosporine can cause ophthalmoplegia, nystagmus, and optic neuropathy. ${ }^{7}$

Episcleritis is the most frequent ocular manifestation and consists in a benign inflammatory reaction in the sclera. It may present with moderate discomfort, erythe$\mathrm{ma}$ in one or both eyes, and diffuse or localized episcleral edema. Compared to other ocular manifestations, episcleritis may be associated with IBD activity. ${ }^{13}$

Scleritis is an inflammation of the sclera that causes eye pain, irradiated towards the face and scalp. Specific for this pathology is the aggravation of symptoms during the night, associating ocular hyperemia and visual loss. ${ }^{13}$

Uveitis is defined as an inflammation of the uveal tract and is currently the third leading cause of irreversible blindness in developed countries. In patients with IBD, it has an insidious onset, is long-lasting, bilateral, and does not correlate with disease activity. ${ }^{13}$

\section{HEPATOBILIARY MANIFESTATIONS}

The hepatic involvement of IBD most often includes PSC. Uncommon hepatobiliary alterations linked with IBD are autoimmune hepatitis/PSC overlap syndrome: IgG-associated cholangiopathy, primary biliary cholangitis, portal vein thrombosis and liver abscess, as well as hepatic amyloidosis, granulomatous hepatitis, and cholelithiasis. The most common liver disease in individuals with IBD that is not correlated with intestinal activity is non-alcoholic fatty liver disease (NAFLD). ${ }^{14}$

The prevalence of PSC varies between $2.4 \%$ and $7.5 \%$ in those with UC and is $3.4 \%$ in patients with CD. ${ }^{15}$ PSC may lead to multiple hepatic and biliary complications including cirrhosis, liver insufficiency, and portal hypertension. Patients with PSC have a high risk of cholangiocarcinoma, and the association between PSC and IBD increases the risk of colorectal cancer. PSC affects the activity and localization of IBD. It is associated with a more extensive form of UC (pancolitis), but with lower rates of activity and colectomy. In patients with CD and PSC, the most common forms are colitis and ileocolitis. ${ }^{16}$

The treatment used in IBD can cause liver toxicity. Methotrexate and thiopurine have the highest risk of hepatotoxicity. Aminosalicylates can rarely cause hepatotoxicity, whereas cases of granulomatous hepatitis and fulminant hepatitis have been reported in the literature. ${ }^{16}$

Since during immunosuppressive treatment reactivation of hepatitis B virus (HBV) is possible, it is necessary to screen patients and vaccinate the seronegative (HBsAg and anti-HBc negative). ${ }^{16}$

\section{PANCREATIC MANIFESTATIONS}

Pancreatic manifestations in subjects with IBD include asymptomatic growth of pancreatic enzymes, acute or chronic pancreatitis, exocrine pancreatic failure, or malignancy. Acute pancreatitis can be favored by gallstones, drugs (mesalazine, thiopurine), duodenal papillary lesions, endoscopic procedural accidents, PSC, and autoimmune pancreatitis. ${ }^{17}$

A recent Danish meta-analysis showed a three-fold risk of developing acute pancreatitis in patients with $\mathrm{CD}$ and a two-fold risk in patients with UC. ${ }^{18}$

The link between IBD and chronic pancreatitis, exocrine pancreatic insufficiency, and pancreatic cancer is not very clear yet, and additional studies are needed. ${ }^{17}$

\section{PULMONARY MANIFESTATIONS}

Pulmonary manifestations occur rarely in patients with IBD. 7 The airways and the gastrointestinal tract have common embryonic origins and a similar structure. The lymphoid tissue present in the mucosa of both organs plays a key 
role. Thus, the mechanisms involved in the development of inflammation are: migration of activated lymphocytes from the gastrointestinal tract to the respiratory tract, the presence of common antigens (bacteria, digestive enzymes) due to loss of intestinal epithelial barrier function, and the presence of antibodies and immune complexes. ${ }^{19}$ Pulmonary manifestations involve the small and large airways, as well as the parenchyma. Extraintestinal complications are drug-induced lung diseases. ${ }^{7}$ Numerous case reports have concluded that respiratory symptoms can be triggered during both periods of IBD activity and remission. ${ }^{19}$

The most common form of EIMs affects the large airways and is more frequently associated with UC. Bronchial inflammation and suppuration are the most common manifestations of lung disease in IBD and include chronic bronchitis and bronchiectasis. The latter is the most commonly reported, with a prevalence of $66 \%$ in patients with large airway involvement. ${ }^{20}$

Lung parenchymal disease is associated with IBD less frequently. The age of onset varies, has a slight female predominance, and is frequently associated with UC. Bronchiolitis obliterans organizing pneumonia (BOOP) is the most common parenchymal manifestation. BOOP can be caused by inhalation or medication injuries and has an acute or subacute onset with fever, cough, dyspnea, and pleuritic chest pain. ${ }^{21}$

The treatment of patients with IBD can cause lung complications. The use of azathioprine (AZA) and 6-mercaptopurine (6-MP) may cause interstitial pneumonia, BOOP, chronic fibrosis/pneumonitis, and pulmonary edema. Long-term use of sulfasalazine and mesalamine may cause eosinophilic pleuritis, eosinophilic pneumonia, and BOOP. Methotrexate may cause the following lung side effects: interstitial pneumonitis, granuloma formation, and bronchiolitis. Biological anti-TNF- $\alpha$ therapy (infliximab, adalimumab, certolizumab) may also cause side effects, requiring close monitoring of therapy. As a result of the suppression of $\mathrm{T}$ cell-mediated immunity, various associated opportunistic infections have been observed, the most common being tuberculosis. Other lung infections associated with biological therapy, especially in the elderly, are: Pneumocystis carinii pneumonia, coccidiomycosis, histoplasmosis, aspergillosis, and actinomycosis. ${ }^{20}$

\section{CARDIOVASCULAR MANIFESTATIONS}

Cardiovascular changes related to IBD include: pericarditis, myocarditis, venous and arterial thromboembolism, heart failure, arrhythmias and conduction disorders, endocarditis, valvulopathy, and Takayasu's arteritis. ${ }^{22}$
The incidence of cardiovascular manifestations is slightly increased compared to the general population. This is supported by a recent cohort study which showed that the prevalence of traditional cardiovascular risk factors is quite low in patients with IBD. ${ }^{23}$ The most common cardiovascular EIM is represented by pericarditis, with a prevalence of $0.19 \%$ in patients with CD and $0.23 \%$ in patients with UC.22,24

The risk of arterial thromboembolic events is increased in patients with IBD, with a similar prevalence in patients with CD and UC. The most common arterial thromboembolic events are acute myocardial infarction, stroke, and mesenteric infarction, with an incidence that is 1.2-, 1.2-, and 3.5-fold higher, respectively, compared to the general population. IBD exacerbations are favorable factors for the occurrence of these events. ${ }^{22}$

Patients with IBD have a two-fold risk of heart failure compared to the control group. When these two conditions coexist, the risk of using systemic corticosteroids is 2.5 times higher. ${ }^{22}$ The incidence of atrial fibrillation in patients with IBD is $11.3 \%$, and the arrhythmic risk increases two-fold during IBD exacerbations compared to the general population.

The association between IBD and endocarditis, mitral and aortic regurgitation and Takayasu's arteritis is very rare. ${ }^{22}$

\section{ANEMIA}

The prevalence of anemia in patients with IBD varies between $6 \%$ and $74 \%$. This very wide variation of prevalence is due to the evaluation criteria used for defining anemia, the type of population investigated (hospitalized /discharged patients, type of IBD, age of patients), but also the evaluation time (at the time of diagnosis, during the disease). ${ }^{25}$

The etiopathogenesis of anemia is multifactorial, but in patients with IBD the most common types of anemia are those due to iron deficiency caused by chronic blood loss, decreased iron absorption, or malnutrition. Other causes that may contribute are vitamin B12 and folate deficiency, as well as the toxic effect of drugs. ${ }^{26}$ Anemia can increase the rate of hospitalizations in patients with IBD and has a negative impact on both quality of life, and cognitive function and work capacity. ${ }^{27}$

\section{IMMUNE THROMBOCYTOPENIC PURPURA}

The presence of immune thrombocytopenic purpura (ITP) in patients with IBD has been described in a limited number of cases. ${ }^{28}$ Researchers support the theory of anti- 
genic mimicry between platelet surface and luminal antigens, thus creating a common immune-mediated pathway to mucosal inflammation and platelet destruction. Studies have shown increased levels of T-helper 1 and CD 4 cells. ${ }^{29}$

\section{RENAL AND GENITOURINARY MANIFESTATIONS}

Patients with IBD have a prevalence of renal impairment of 4-23\%.7 Renal manifestations include nephrolithiasis, glomerulonephritis, tubulointerstitial nephritis, and amyloidosis. ${ }^{30}$ The most common form of glomerulopathy in patients with IBD is IgA nephropathy, which is associated with HLA-DR1. Tubulointerstitial nephritis may be a druginduced complication of IBD caused by the administration of 5-aminosalicylic acid and sulfasalazine. ${ }^{7}$ Nephrolithiasis affects $5-15 \%$ of patients with IBD, predominantly those with $\mathrm{CD}$ and ileo-colonic involvement. The most common stones are those of calcium oxalate (by malabsorption of bile acids) and those of uric acid (by decreasing urinary $\mathrm{pH}$ and urinary volumes).?

A recent cohort study (SIBDCS) on 2,323 patients showed that intestinal surgery, especially ileal/ileo-colonic surgery, may increase the incidence of kidney stones. ${ }^{31}$

A recent study on genital manifestations showed that the prevalence of cervical dysplasia and HPV 16/18 infection was significantly higher than in the control group. ${ }^{32}$

\section{DEPRESSION AND ANXIETY DISORDERS}

Anxiety disorders and depression are more common in patients with IBD compared to the general population. This relationship has not been fully elucidated, but the suggested mechanisms include activation of the inflammatory response in the brain, compromised integrity of the bloodbrain barrier, and the impact of the intestinal microbiota. ${ }^{33}$ A Canadian study on 6,000 patients with IBD showed that the prevalence and incidence of depression, anxiety, and bipolar disorders were significantly higher compared to the general population. ${ }^{33}$

A recent analysis of non-pharmacological therapy that included 10 studies showed that cognitive behavioral therapy, as well as attention-based and solution-based therapy significantly contributed to the reduction of anxiety and depression in adults compared to control groups. ${ }^{34}$

\section{NEUROLOGICAL MANIFESTATIONS}

The most common neurological manifestations in patients with IBD are peripheral neuropathies, unrelated to intesti- nal activity. In patients with $\mathrm{CD}$ and ileocecal resection, a typical neurological complication is found, namely peripheral polyneuropathy due to vitamin B12 deficiency. ${ }^{35}$

Focal intracerebral lesions of the white matter were detected in $42 \%$ of patients with CD and in $46 \%$ of patients with UC. Recurrent facial paralysis associated with Melkerssson-Rosenthal syndrome has been specifically observed in some patients with CD. ${ }^{7}$

Anti-TNF- $\alpha$ therapy is associated with Guillain-Barré syndrome and chronic inflammatory demyelinating polyneuropathy, while metronidazole and sulfasalazine may induce peripheral neuropathy. ${ }^{36}$

\section{CONCLUSIONS}

In our extensive analysis we highlighted that extraintestinal manifestations of IBD are very frequent, can affect almost any organ system, and have the potential to become more debilitating than the intestinal disease itself. We therefore draw attention to the importance of proper recognition and treatment of extraintestinal manifestations of IBD that will help reduce patient morbidity and mortality, as well as improve quality of life.

\section{CONFLICT OF INTEREST}

Nothing to declare.

\section{REFERENCES}

Zhao J, Lu Q, Liu Y, et al. Th17 Cells in Inflammatory Bowel Disease: Cytokines, Plasticity, and Therapies. Journal of Immunology Research. 2021;1-14.

2. Jang HJ, Kang B, Choe BH. The difference in extraintestinal manifestations of inflammatory bowel disease for children and adults. Trans/ Pediatr. 2019;8:4-15.

3. Martinez-Fierro ML, Garza-Veloz I, Rocha-Pizaña MR, et al. Serum cytokine, chemokine, and growth factor profiles and their modulation in inflammatory bowel disease. Medicine (Baltimore). 2019;98:e17208.

4. Kim JM, Cheon JH. Pathogenesis and clinical perspectives of extraintestinal manifestations in inflammatory bowel diseases. Intest Res. 2020;18:249-264.

5. Ma C, Vasu R, Zhang H. The Role of Long-Chain Fatty Acids in Inflammatory Bowel Disease. Mediators Inflamm. 2019;2019: 8495913.

6. Malaty HM, Lo GH, Hou JK. Characterization and prevalence of spondyloarthritis and peripheral arthritis among patients with inflammatory bowel disease. Clin Exp Gastroenterol. 2017;10:259-263.

7. Chams S, Badran R, Sayegh SE, Chams N, Shams A, Hajj Hussein I. Inflammatory bowel disease: Looking beyond the tract. Int J Immunopathol Pharmacol. 2019:33:1-18.

8. Kotze LM, Costa CT, Cavassani MF, Nisihara RM. Alert for bone alterations and low serum concentrations of vitamin $D$ in patients with intestinal inflammatory disease. Rev Assoc Med Bras. 2017;63:13-17.

9. Ungureanu L, Cosgarea R, Alexandru Badea M, et al. Cutaneous manifestations in inflammatory bowel disease (Review). Exp Ther Med. 2020;20:31-37.

10. Antonelli E, Bassotti G, Tramontana M, et al. Dermatological Manifestations in Inflammatory Bowel Diseases. J Clin Med. 2021;10;1-16.

11. Lauritano D, Boccalari E, Di Stasio D, et al. Prevalence of Oral Lesions and Correlation with Intestinal Symptoms of Inflammatory Bowel Disease: A Systematic Review. Diagnostics (Base/). 2019;9:1-16. 
12. Kumar KM, Nachiammai N, Madhushankari GS. Association of oral manifestations in ulcerative colitis: A pilot study. J Oral Maxillofac Pathol. 2018;22:199-203.

13. Troncoso LL, Biancardi AL, de Moraes HV Jr, Zaltman C. Ophthalmic manifestations in patients with inflammatory bowel disease: a review. World J Gastroenterol. 2017;23:5836-5848.

14. Silva J, Brito BS, de N. Silva IN, et al. Frequency of Hepatobiliary Manifestations and Concomitant Liver Disease in Inflammatory Bowel Disease Patients. Biomed Res Int. 2019;2019:7604939.

15. de Vries AB, Janse M, Blokzijl H, Weersma RK. Distinctive inflammatory bowel disease phenotype in primary sclerosing cholangitis. World $\mathrm{J}$ Gastroenterol. 2015;21:1956-1971.

16. Fousekis FS, Theopistos VI, Katsanos $\mathrm{KH}$, et al. Hepatobiliary Manifestations and Complications in Inflammatory Bowel Disease: A Review. Gastroenterol Res. 2018;11:83-94.

17. lida T, Wagatsuma K, Hirayama D, Yokoyama Y, Nakase H. The Etiology of Pancreatic Manifestations in Patients with Inflammatory Bowel Disease. $J$ Clin Med. 2019;8:916.

18. Fousekis FS, Theopistos VI, Katsanos KH, Christodoulou DK. Pancreatic Involvement in Inflammatory Bowel Disease: A Review. J Clin Med Res. 2018;10:743-751.

19. Kacprzak A, Szturmowicz M, Kus J. Respiratory system involvement in inflammatory bowel diseases. Adv Respir Med. 2017;85:161-168.

20. Ji XQ, Wang LX, Lu DG. Pulmonary manifestations of inflammatory bowel disease. World J Gastroenterol. 2014;20:13501-13511.

21. Xu L, Xiao W, Ma D, Zhou S, Zhang Q. Ulcerative colitis combined with acute interstitial lung disease and airway disease: A case report and literature review. Exp Ther Med. 2014;8:1229-1236.

22. Bunu DM, Timofte CE, Ciocoiu M, et al. Cardiovascular Manifestations of Inflammatory Bowel Disease: Pathogenesis, Diagnosis, and Preventive Strategies. Gastroenterol Res Pract. 2019;2019:3012509.

23. Harbord M, Annese V, Vavricka SR, et al. The First European Evidencebased Consensus on Extra-intestinal Manifestations in Inflammatory Bowel Disease. J Crohns Colitis. 2016;10:239-254.

24. Mitchell NE, Harrison N, Junga Z, Singla M. Heart Under Attack: Cardiac
Manifestations of Inflammatory Bowel Disease. Inflamm Bowel Dis. 2018:24:2322-2326.

25. Ali SA, Abbasi Z, Shahid B, et al. The prevalence, characteristics, and determinants of anaemia in newly diagnosed patients with inflammatory bowel disease. Gastroenterology Rev. 2019;14:39-47.

26. Tulewicz-Marti E, Moniuszko A, Rydzewska G. Management of anemia in inflammatory bowel disease: a challenge in everyday clinical practice. Gastroenterol Rev. 2017;12:239-243.

27. Nemes RM, Pop CS, Calagiu D, et al. Anemia in inflammatory bowel disease more than an extraintestinal complication. Rev Med Chir. 2016;120:34-39.

28. Epistola R, Do T, Vankina R, et al. Immune thrombocytopenic purpura (ITP) as an uncommon extraintestinal complication of Crohn's disease: case vignette and systematic literature review. Case Rep Hematol. 2020;2020:4785759

29. Jimenez K, Kulnigg-Dabsch S, Gasche C. Management of Iron Deficiency Anemia. Gastroenterol Hepatol (N Y). 2015;11:241-250.

30. Fagagnini S, Heinrich $H$, Rossel JB, et al. Risk factors for gallstones and kidney stones in a cohort of patients with inflammatory bowel diseases. PLOS ONE. 2017;12:e0185193.

31. Pittet $V$, Juillerat $P$, Mottet $C$, et al. Cohort Profile: The Swiss Inflammatory Bowel Disease Cohort Study (SIBDCS). Int J Epidemiol. 2009;38:922-931.

32. Li M, Yang QF, Cao Q, et al. High-risk human papilloma virus infection and cervical neoplasm in female inflammatory bowel disease patients: a crosssectional study. Gastroenterol Rep (Oxf). 2019;7:338-344.

33. Kuźnicki P, Kempiński R, Neubauer K. The emerging role of mood disorders in inflammatory bowel diseases. Adv Clin Exp Med. 2020;29:1505-1510.

34. Davis SP, Bolin LP, Crane PB, Crandell J. Non-pharmacological Interventions for Anxiety and Depression in Adults With Inflammatory Bowel Disease: A Systematic Review and Meta-Analysis. Front Psychol. 2020;11:538741.

35. Singh S, Kumar N, Loftus EV Jr, Kane SV. Neurologic complications in patients with inflammatory bowel disease: increasing relevance in the era of biologics. Inflamm Bowel Dis. 2013;19:864-872.

36. Ott C, Schölmerich J. Extraintestinal manifestations and complications in IBD. Nat Rev Gastroenterol Hepatol. 2013;10:585-595. 